\title{
DUAL-POLARIMETRIC AGRICULTURAL CHANGE ANALYSIS OF LONG BASELINE TANDEM-X TIME SERIES DATA
}

\author{
Alberto Alonso-González ${ }^{1}$, Hannah Joerg ${ }^{1}$, Kostas Papathanassiou ${ }^{1}$, and Irena Hajnsek ${ }^{1,2}$ \\ ${ }^{1}$ German Aerospace Center (DLR), Germany; e-mail: alberto.alonso-gonzalez@dlr.de \\ ${ }^{2}$ Institute of Environmental Engineering, ETH Zurich, Switzerland
}

\begin{abstract}
The standard TanDEM-X baselines have been designed to optimize the high resolution global Digital Elevation Model (DEM) generation. However, during the Science Phase of the mission longer baselines are available. This allows interferometric measurements with a higher vertical sensitivity, more appropriate for agricultural applications, where the crop heights are too small to be properly detected and analyzed with the standard baselines. This paper evaluates the use of the experimental long baseline TanDEM-X acquisitions for the monitoring of the agricultural changes in dual-pol singlepass interferometric time series.
\end{abstract}

Index Terms - SAR, Polarimetry, TanDEM-X, PolInSAR, time series

\section{INTRODUCTION}

TanDEM-X (TerraSAR-X add-on for Digital Elevation Measurements) is an innovative spaceborne radar single-pass interferometer, based on two satellites flying in a close HELIX formation [1]. It allows the acquisition of bistatic SAR data at X-band, with short along-track baselines and a wide range of possible cross-track baselines, in order to demonstrate and investigate single-pass interferometry from space.

The primary objective of the TanDEM-X mission is to generate a high-resolution global Digital Elevation Model (DEM) of the Earth. Consequently, the nominal baselines of the HELIX formation were selected according to this objective, with a height of ambiguity in the order of 30 to 45 meters [2]. These baselines, however, are not optimal for agricultural applications, where larger baselines would be more appropriate for monitoring agricultural vegetation with interferometric techniques. In this case, a height of ambiguity of around 5 to 10 meters would be more convenient. As mentioned in [1], large baselines on this order may be employed with TanDEM-X, since only $5 \%$ of the critical baseline length is used during the nominal DEM data acquisition. In order to demonstrate these capabilities, a long baseline experimental phase has been planned and implemented in the Science

This work has been conducted under the funding of the HGF Alliance HA-310 'Remote Sensing and Earth System Dynamics'.
Phase. This paper investigates the use of dual-pol long baseline TanDEM-X time series acquired during this phase for monitoring agricultural vegetation.

\section{SCIENCE PHASE MISSION}

The TanDEM-X Science Phase lasted 15 months, started in October 2014 and ended in December 2015. During this time, two main operations modes have been employed: the pursuit monostatic and the bistatic mode. In the pursuit monostatic phase, the along-track baseline is $76 \mathrm{~km}$ and the two satellites are operated independently from each other. This phase lasted from October 2014 to February 2015, and may be employed to apply two-pass interferometry with very small temporal baselines.

In the bistatic phase either TSX or TDX transmitted while both satellites received simultaneously. In this mode, the along-track baseline was reduced to less than a kilometer during the commissioning phase in March 2015. From April to August 2015, large cross-track baselines were maintained, up to 3 to $4 \mathrm{~km}$ on the equator, decreasing at higher latitudes. Finally, in September 2015, the orbits were changed back to the nominal cross-track shorter baselines, in the order of $0-250$ meters.

\section{LONG BASELINE TIME SERIES}

The larger baselines employed in this experimental phase increase significantly the height accuracy of generated DEMs but also reduce the height of ambiguity, making these acquisitions not practical for DEM generation over areas with significant topography. Moreover, volume decorrelation is also larger, reducing significantly the interferometric coherence over forested areas. This paper analyzes the use of this data for monitoring agricultural vegetation, taking profit of the improved height accuracy for the determination and monitoring of different crop parameters.

For this analysis, a time series dataset from an agricultural testsite located in Wallerfing, in southern Germany, will be considered. Table 1 shows the set of employed dual-pol TanDEM-X long baseline acquisitions over this area. Two 
time series are available in different combinations of incident angles, baselines and with ascending and descending passes. These time series cover most of the growing season of crops as corn, wheat, barley, sugar beet and rape. In parallel to the SAR acquisitions, ground measurements were performed, including land-use, vegetation height, soil-moisture and plant water content.

Fig. 1 shows the $\mathrm{HH}$ amplitude and coherence images for the acquisition performed on the 25th of May. The upper part of the image, marked with a red square, corresponds to the mentioned Wallerfing agricultural testsite.

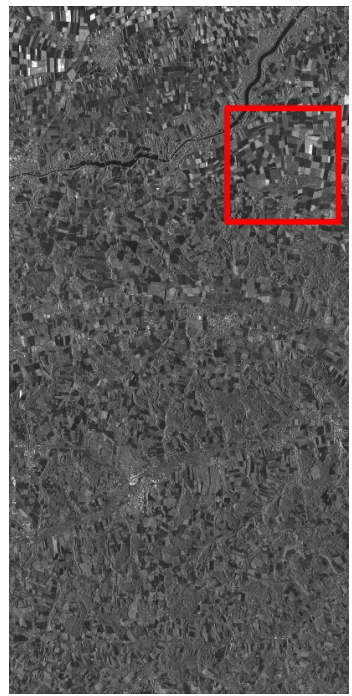

(a) Amplitude $\mathrm{HH}$

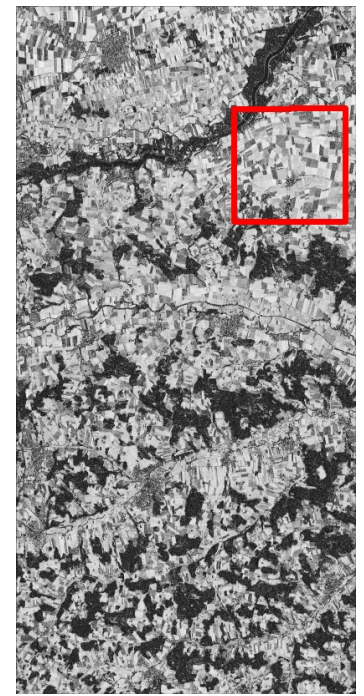

(b) Coherence $\mathrm{HH}$
Fig. 1: Amplitude and coherence for the $\mathrm{HH}$ polarization of the acquisition of 25th May. Coherence is scaled from 0 (black) to 1 (white).

As it may be seen in Fig. 1b, the coherence is almost completely lost in the forested area near the river in the upper part of the image, due to the high volume decorrelation at X-band with this long baseline. However, in the agricultural fields below the river, high values of coherence are still obtained.

\section{POLARIMETRIC CHANGE ANALYSIS}

This data basis allows to investigate the potential of polarimetric and interferometric change analysis for determining the changes in agriculture vegetation development in the dualpol space at X-band. To perform this study, the polarimetric change analysis described in [3] is adapted to the dual-pol case and applied to the dataset. In this case, the scattering vector measured by the radar may be expressed as

$$
\mathbf{k}=\left[S_{h h}, S_{v v}\right]^{T}
$$

where $S_{h h}$ and $S_{v v}$ represent the scattering coefficient for horizontal and vertical transmitted and received polarizations.
Under the assumption of distributed scattering, the PolSAR response of a target may be completely characterized by the second order statistics of the scattering vector $\mathbf{k}$, that is, the covariance matrix, which may be estimated as

$$
\mathbf{Z}=\left\langle\mathbf{k k}^{H}\right\rangle_{n}=\frac{1}{n} \sum_{i=1}^{n} \mathbf{k}_{i} \mathbf{k}_{i}^{H}
$$

where $\mathbf{k}_{i}$ represents the scattering vector of the $i$-th pixel, as defined in (1), and $n$ represents the number of independent pixels averaged.

The polarimetric change between two acquisitions, characterized by polarimetric responses $\mathbf{Z}_{1}$ and $\mathbf{Z}_{2}$, may be obtained by exploiting the differences between covariance matrices. In [4] the polarimetric contrast measure was introduced, in order to describe the relative change of the backscattered power at each polarization state $\mathbf{w}$

$$
P_{c}\left(\mathbf{Z}_{1}, \mathbf{Z}_{2}, \mathbf{w}\right)=\frac{\mathbf{w}^{H} \mathbf{Z}_{2} \mathbf{w}}{\mathbf{w}^{H} \mathbf{Z}_{1} \mathbf{w}}
$$

This concept is exploited in [3] to define a polarimetric change analysis technique. The generalized eigendecomposition of $\mathbf{Z}_{1}$ and $\mathbf{Z}_{2}$ matrices is employed to extract the range of values of the polarimetric contrast and the corresponding polarization states w. Moreover, a change representation is proposed for the interpretation of the observed changes. This representation classifies the observed changes according to the increase or decrease of (3), and depicts the polarization states that produce the change in two images

$$
\begin{aligned}
& \mathbf{p}_{\text {inc }}=10\left[\sum_{i \mid \lambda_{i}>1}^{p}\left(\log _{10} \lambda_{i} \mathbf{p}_{i}\right)^{2}\right]^{\frac{1}{2}} \\
& \mathbf{p}_{\text {dec }}=10\left[\sum_{i \mid \lambda_{i}<1}^{p}\left(-\log _{10} \lambda_{i} \mathbf{p}_{i}\right)^{2}\right]^{\frac{1}{2}},
\end{aligned}
$$

where $\mathbf{p}_{\text {inc }}$ and $\mathbf{p}_{\text {dec }}$ stand for the polarimetric change representation of the increase and decrease, respectively, $\lambda_{i}$ represent the generalized eigenvalues and $\mathbf{p}_{i}$ is the RGB representation of the generalized eigenvectors, as described in [3]. In the dual-pol case, only red and green components are employed, corresponding to $\mathrm{HH}$ and VV polarizations, respectively.

In these representations the color describes the type of change whereas the intensity represents the amount of change, i.e. increase or decrease. Note that this approach is able to determine and discriminate not only the amount of change, but also the type of change, i.e. the scattering mechanisms associated to the change. Fig. 2 shows the change matrices representing the mentioned change analysis representation for the two time series shown in Table 1. The upper diagonal represents the increase $\mathbf{p}_{\text {inc }}$ while the lower the decrease $\mathbf{p}_{d e c}$. In this case the interpretation of the changes is 


\begin{tabular}{cccccc}
\hline \hline Acquisition Date & Transmitting Sat. & Pass & Incidence angle & Eff. Baseline $(\mathrm{m})$ & Height of ambiguity (m) \\
\hline $06 / 05 / 2015$ & TerraSAR-X & Descending & 31.91 & 976 & 5.19 \\
$17 / 05 / 2015$ & TerraSAR-X & Descending & 31.94 & 978 & 5.19 \\
28/05/2015 & TerraSAR-X & Descending & 31.94 & 980 & 5.18 \\
$08 / 06 / 2015$ & TanDEM-X & Descending & 32.13 & 983 & 5.20 \\
$11 / 07 / 2015$ & TanDEM-X & Descending & 32.13 & 985 & 5.19 \\
$02 / 08 / 2015$ & TanDEM-X & Descending & 32.13 & 984 & 5.19 \\
\hline 25/05/2015 & TanDEM-X & Ascending & 44.20 & 981 & 7.86 \\
$05 / 06 / 2015$ & TanDEM-X & Ascending & 44.20 & 979 & 7.87 \\
$16 / 06 / 2015$ & TanDEM-X & Ascending & 44.20 & 981 & 7.84 \\
$30 / 07 / 2015$ & TanDEM-X & Ascending & 44.20 & 982 & 7.87 \\
$10 / 08 / 2015$ & TanDEM-X & Ascending & 44.20 & 979 & 7.87 \\
$21 / 08 / 2015$ & TanDEM-X & Ascending & 44.20 & 980 & 7.87 \\
$01 / 09 / 2015$ & TanDEM-X & Ascending & 44.20 & 979 & \\
\hline \hline
\end{tabular}

Table 1: List of employed dual-pol HHVV TanDEM-X long baseline acquisitions over the Wallerfing testsite.

complex due to the limited penetration of the X-band radar signal into the crops. However, the starting of the growing process of the maize may be seen in the first acquisitions, from May to the start of June, appearing as a yellowish spot. After this point, even when the plants continue growing, the signal backscattered corresponds only to the interaction with the top part of the canopy and no significant changes are perceived.

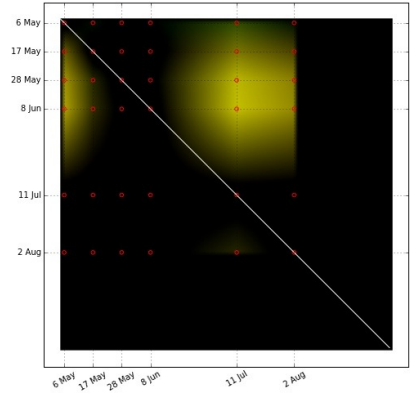

(a) Maize 1st series

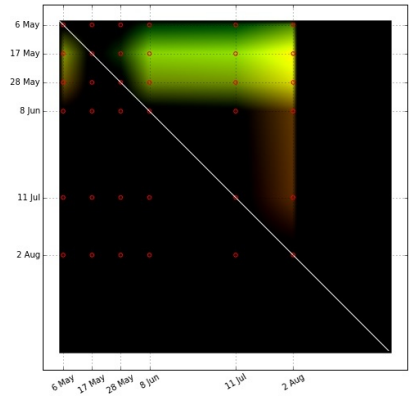

(c) Wheat $1 \mathrm{st}$ series

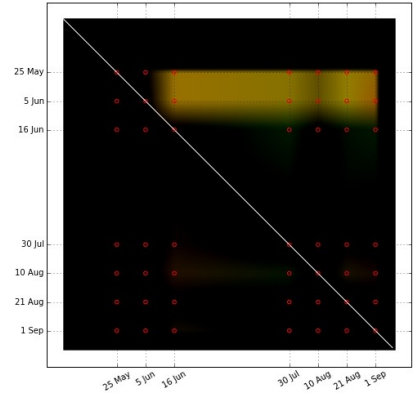

(b) Maize 2nd series

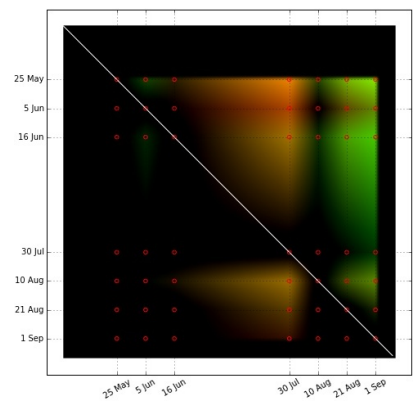

(d) Wheat 1 st series
Fig. 2: Change matrices for the two time series over fields corresponding to maize and wheat.

In the wheat crops, in Figs. 2c and 2d, two main changes are seen, in green and red colors. These changes correspond to the plant growing during May and the harvest at the end of July, respectively. Still, the interpretation of these changes may be difficult employing only the change matrices.

\section{INTERFEROMETRIC HEIGHT DIFFERENCE}

Additionally, single-pass interferometric measurements can also be employed in order to extend the polarimetric information and estimate the changes in the crop height, for instance, which is a very relevant aspect for agricultural monitoring [5]. In this case, due to the long baseline acquisitions, an improved vertical sensitivity is obtained. It is worth mentioning that the changes in the crop height are not directly measured through interferometry. Instead, the changes on the radar scattering phase center are detected, which are expected to be directly related to changes in the crop height, among other parameters.

Fig. 3 shows the estimated height difference over an area of the image corresponding to the Wallerfing test site. In this case the second time series presented in Table 1 has been employed, due to the larger amount of acquisitions. Fig. 3a presents the crop map of the area, with maize, wheat and rape fields in colors yellow, blue and red, respectively. Figs. 3b-3d show the height difference with respect to the first acquisition, on the 25th May. In these results, the areas with low coherence (below 0.3) have been masked out.

As it may be seen in Fig. 3b, no significant height changes are observed during these two acquisitions that are only separated by 11 days. When increasing the time span, in Fig. 3c, from 25th May to 30th July, significant changes may be observed in the obtained height. The maize crops have grown notably during these dates, appearing in reddish color. On the other hand, a decrease is observed in the interferometric height over the rape fields, which is produced due to the harvest of these fields at the end of July. Over the wheat fields the changes are not as notable as for the other fields, since the maximum height of these crops is around 1 meter. However, a small decrease may also be observed as these crops are also harvested at similar dates. Fig. $3 d$ shows the height differ- 


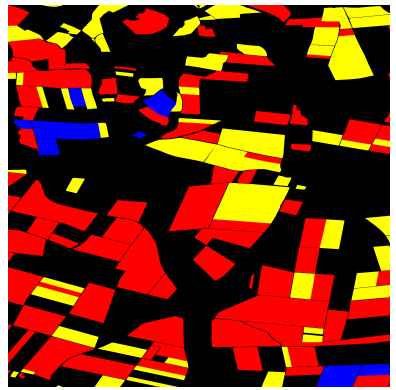

(a) Crop map

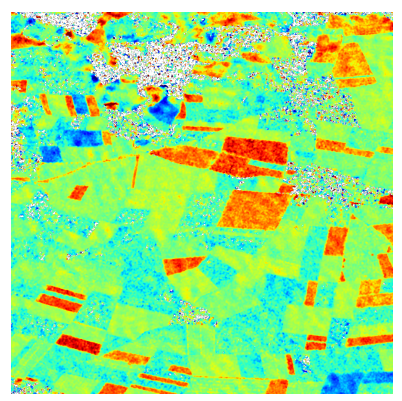

(c) Height diff 30th July

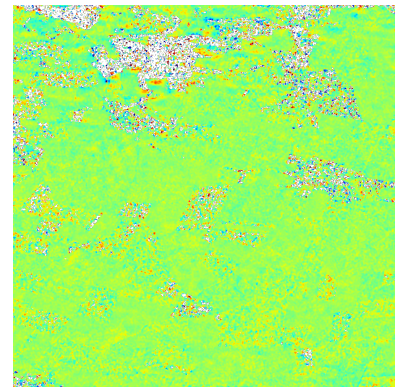

(b) Height diff 5th June

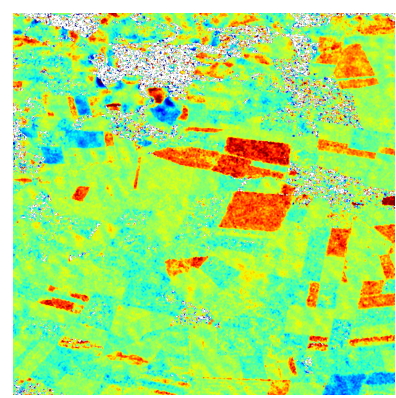

(d) Height diff 1st September

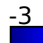

m

Fig. 3: Crop map (a) of the selected area (yellow: maize, blue: rape, red: wheat), and interferometric height differences (b)(d) with respect to the acquisition in 25th May.

ences from 25th May to 1st September, showing the consistency of the previously mentioned height changes.

Fig. 4 shows the height evolution of one field for each type of crop. In this case, the measure is performed at a field level and the polarimetric space is explored in order to extract the maximum and minimum height in the coherence region. These height differences are represented in red and blue colors, respectively. All the plots are differential measurements with respect to the first acquisition, on the 25th May, represented with a 0 value. As it may be seen, the height increase of the maize due its grown and the decrease in rape and maize crops is clearly visible in these plots.

\section{CONCLUSIONS}

This paper has shown the usefulness of TanDEM-X dual-pol long baseline acquisitions for the monitoring of agricultural areas. The polarimetric and interferometric information may be combined to detect the changes of the different fields and monitor the crop phenology and evolution over large areas from space. For instance, the plant growing and harvest can be clearly detected and even the height changes of the maize crops, in the order of a meter, can be properly detected.

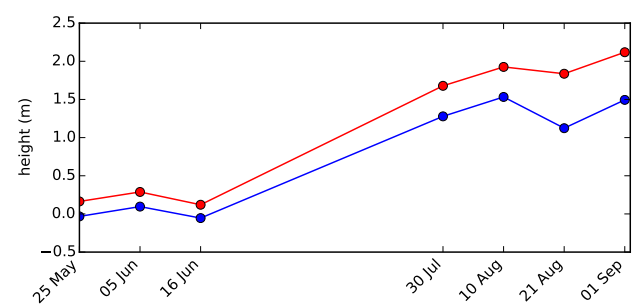

(a) Maize

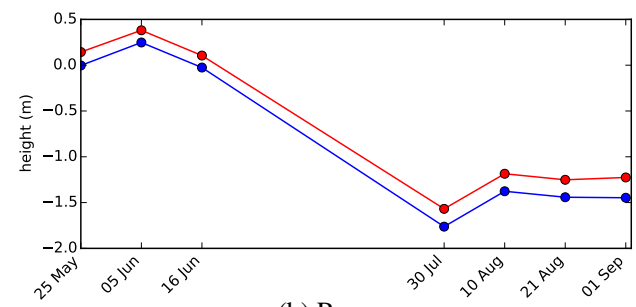

(b) Rape

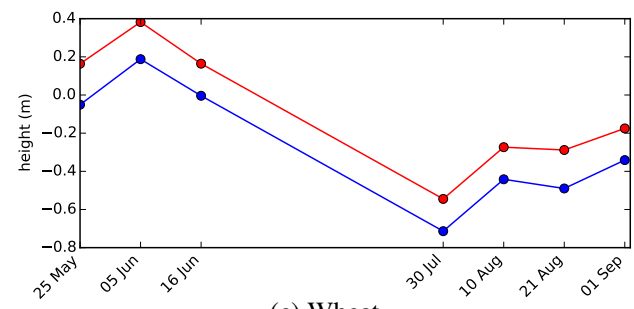

(c) Wheat

Fig. 4: Interferometric height differences evolution for different crops. The maximum and minimum height in the coherence region are marked in red and blue colors, respectively.

\section{REFERENCES}

[1] G. Krieger, A. Moreira, H. Fiedler, I. Hajnsek, M. Werner, M. Younis, and M. Zink, "TanDEM-X: A Satellite Formation for High-Resolution SAR Interferometry," Geoscience and Remote Sensing, IEEE Transactions on, vol. 45, no. 11, pp. 3317-3341, Nov 2007.

[2] G. Krieger, H. Fiedler, I. Hajnsek, M. Eineder, M. Werner, and A. Moreira, "TanDEM-X: mission concept and performance analysis," in Geoscience and Remote Sensing Symposium, 2005. IGARSS '05. Proceedings. 2005 IEEE International, July 2005, vol. 7, pp. 4890-4893.

[3] A. Alonso-Gonzalez, T. Jagdhuber, and I. Hajnsek, "Exploitation of Agricultural Polarimetric SAR time series with Binary Partition Trees," Proc. ESA PolInSAR, 2015.

[4] A. Kostinski and W-M Boerner, "On the polarimetric contrast optimization," Antennas and Propagation, IEEE Transactions on, vol. 35, no. 8, pp. 988-991, Aug 1987.

[5] C. Rossi and E. Erten, "Paddy-Rice Monitoring Using TanDEM-X," Geoscience and Remote Sensing, IEEE Transactions on, vol. 53, no. 2, pp. 900-910, Feb 2015. 